Article

\title{
Recurrence Relations for Orthogonal Polynomials on Triangular Domains
}

\author{
Abedallah Rababah \\ Department of Mathematics, Jordan University of Science and Technology, Irbid 22110, Jordan; \\ rababah@just.edu.jo; Tel.: +962-02-720-1000 \\ Academic Editors: Zhongqiang Zhang and Mohsen Zayernouri \\ Received: 26 December 2015; Accepted: 7 April 2016; Published: 12 April 2016
}

\begin{abstract}
In Farouki et al, 2003, Legendre-weighted orthogonal polynomials $\mathcal{P}_{n, r}(u, v, w), r=$ $0,1, \ldots, n, n \geq 0$ on the triangular domain $T=\{(u, v, w): u, v, w \geq 0, u+v+w=1\}$ are constructed, where $u, v, w$ are the barycentric coordinates. Unfortunately, evaluating the explicit formulas requires many operations and is not very practical from an algorithmic point of view. Hence, there is a need for a more efficient alternative. A very convenient method for computing orthogonal polynomials is based on recurrence relations. Such recurrence relations are described in this paper for the triangular orthogonal polynomials, providing a simple and fast algorithm for their evaluation.
\end{abstract}

Keywords: recurrence relation; bivariate orthogonal polynomials; Bernstein polynomials; Legendre polynomials; triangular domains

MSC: 65Dxx, 33C45

\section{Introduction}

Orthogonal polynomials are very strong tools in approximation theory and play a fundamental role in finding the least-squares approximation in explicit form. They are well-studied, and a lot of research has been done for the univariate case, see [1]. Orthogonal polynomials over a square region can be constructed using the tensor product of univariate orthogonal polynomials, see [2,3]. Orthogonal polynomials over triangular domains have to be determined in a different way, see [4]. A scheme for orthogonal bivariate polynomials on triangular regions is constructed in [5]. The Legendre weight function $W(u, v, w)=1$ is used; these Legendre-weighted orthogonal polynomials are given in the Bernstein basis form, in order to take full advantage of the numerical stability property of the later basis. In [5], to find these bivariate orthogonal polynomials on triangular domains, the sums have to be expanded, and many multiplications have to be performed. This leads to a time-consuming and costly method of construction. There is an essential need for an easy method to find these orthogonal polynomials. It is known that the most convenient method to compute orthogonal polynomials is using a recurrence relation. For the case under consideration, such an algorithm is described in this paper in Section 6.

Connections between bivariate Bernstein and Jacobi bases are considered in [6] without considering the recurrence relations for these polynomials. Orthogonal polynomials on the interior of the triangle based on second-order linear partial differential equations is considered in [7]. The approach is different when considering the boundary of the triangle which needs applying the directional derivatives.

This paper is organized as follows. The univariate Legendre and Bernstein polynomials are introduced in Section 2. In Sections 3 and 4, bivariate polynomials and bivariate polynomials on triangular domains are introduced. Legendre-weighted orthogonal polynomials are given in Section 5, and their recurrence relations are given in Section 6. We end this paper with conclusions in Section 7. 


\section{Univariate Legendre and Bernstein Polynomials}

The univariate Legendre polynomials, $L_{n}(x)$, of degree $n$ are orthogonal on the interval $[-1,1]$ with respect to the weight function $W(x)=1$. They are traditionally defined over $[-1,1]$ to highlight their symmetry, while the Bernstein polynomials, $B_{i}^{n}(u)$, are defined over $[0,1]$ to highlight their geometric behaviour. Since the final results will be given in Bernstein form, the suitable interval for both polynomials is $[0,1]$. In this case, the shifted Legendre polynomials $L_{n}\left(\frac{x+1}{2}\right)=: L_{n}(u), u \in[0,1]$ are used.

For $u \in[0,1]$, the Bernstein polynomial basis is defined as, see [8],

$$
b_{i}^{n}(u)=\left(\begin{array}{c}
n \\
i
\end{array}\right) u^{i}(1-u)^{n-i}, \text { where }\left(\begin{array}{c}
n \\
i
\end{array}\right)=\frac{n !}{i !(n-i) !}, i=0,1, \ldots, n .
$$

For $u \in[0,1]$, the univariate Legendre polynomials satisfy the following three term recurrence relation, see [1]:

$$
L_{n}(u)=\frac{2 n-1}{n}(2 u-1) L_{n-1}(u)-\frac{n-1}{n} L_{n-2}(u), L_{0}(u)=1, L_{1}(u)=2 u-1, n \geq 2 .
$$

The polynomials $L_{n}$ satisfy the following orthogonality conditions:

$$
\int_{0}^{1} L_{n}(u) L_{m}(u) d u=\left\{\begin{array}{ll}
\frac{1}{2 n+1}, & \text { if } m=n \\
0, & \text { if } m \neq n
\end{array} .\right.
$$

\section{Bivariate Orthogonal Polynomials}

Let $G \subseteq \mathbb{R}^{2}$ be a finite simply connected domain bounded by a curve $\Gamma$. A function $W(x, y) \geq 0$ is called a weight function over $G$ if $\iint_{G} W(x, y) d x d y$ exists and is positive. The weight function $W$ induces a function space $L_{2}(W, G)$ with the inner product:

$$
\langle f, g\rangle=\iint_{G} W(x, y) f(x, y) g(x, y) d x d y .
$$

The integrals

$$
W_{n, i}=\iint_{G} W(x, y) x^{n-i} y^{i} d x d y
$$

are finite and called moments of $W$. Similar to the univariate case, weight functions are used to define bivariate orthogonal polynomials. A system of polynomials $P_{n, i}(x, y), n \geq 0, i=0,1, \ldots n$ is called orthogonal over $G$ with respect to the weight function $W(x, y)$ if all the leading coefficients $c_{n, i}$ of $P_{n, i}(x, y)$ are positive and

$$
\iint_{G} W(x, y) P_{n, i}(x, y) P_{m, j}(x, y) d x d y=\delta_{n, m} \delta_{i, j}
$$

where $\delta$ is the Kronecker delta, see $[9,10]$. Like the univariate case, for any weight function $W$ over a domain $G$ there exists a system of orthogonal polynomials. Up to this normalization, the system of orthogonal polynomials is unique. For $n \geq 1$, we define the space $\mathcal{L}_{n}$ of polynomials of degree $n$ that are orthogonal to all polynomials of degree $<n$ over the domain $G$, i.e.,

$$
\mathcal{L}_{n}=\left\{P \in \Pi_{n}: \quad P \perp \Pi_{n-1}\right\},
$$


and $\Pi_{n-1}$ to be the space of all polynomials of degree $n-1$ over the domain $G$. These polynomials are usually represented in a triangular table:

$$
\begin{aligned}
& P_{0,0}(x, y) \\
& P_{1,0}(x, y), \quad P_{1,1}(x, y) \\
& P_{2,0}(x, y), \quad P_{2,1}(x, y), \quad P_{2,2}(x, y) \\
& P_{n, 0}(x, y), \quad P_{n, 1}(x, y), \quad P_{n, 2}(x, y), \quad \ldots, \quad P_{n, n}(x, y)
\end{aligned}
$$

The $k$ th row of this triangular table contains $k+1$ bivariate orthogonal polynomials. Thus, for total degree $n$, there is a total of $(n+1)(n+2) / 2$ bivariate orthogonal polynomials, see [11-13].

\section{Bivariate Polynomials on Triangular Domains}

The bivariate polynomials on triangular domains are used in design and modelling in Computer Aided Geometric Design; they are defined using the barycentric coordinates. Let $\mathbf{p}_{k}=\left(x_{k}, y_{k}\right)$, $k=1,2,3$ be the vertices (non-collinear) of a triangle $T$ and $\mathbf{p}$ be a point, all in the plane. It is always possible to express $\mathbf{p}$ as a barycentric combination of $\mathbf{p}_{k}, k=1,2,3$ in the form $\mathbf{p}=u \mathbf{p}_{1}+v \mathbf{p}_{2}+w \mathbf{p}_{3}$, where $u, v, w \geq 0, u+v+w=1$, see [14] and the references therein. Using Cramer's rule, the barycentric coordinates are given as the ratio of areas of subtriangles of the base triangle in explicit form:

$$
u=\frac{\operatorname{arca}\left(\mathbf{p}, \mathbf{p}_{2}, \mathbf{p}_{3}\right)}{\operatorname{area}\left(\mathbf{p}_{1}, \mathbf{p}_{2}, \mathbf{p}_{3}\right)}, \quad v=\frac{\operatorname{area}\left(\mathbf{p}_{1}, \mathbf{p}, \mathbf{p}_{3}\right)}{\operatorname{area}\left(\mathbf{p}_{1}, \mathbf{p}_{2}, \mathbf{p}_{3}\right)}, \quad w=\frac{\operatorname{area}\left(\mathbf{p}_{1}, \mathbf{p}_{2}, \mathbf{p}\right)}{\operatorname{area}\left(\mathbf{p}_{1}, \mathbf{p}_{2}, \mathbf{p}_{3}\right)}
$$

Consider the triangular region $T$ that is given by the barycentric coordinates as follows

$$
T=\{(u, v, w): u, v, w \geq 0, u+v+w=1\}
$$

and the triples $\alpha=(i, j, k)$ of non-negative integers with $|\alpha|=i+j+k$. A system of polynomials $\left\{\mathcal{P}_{n, r}(u, v, w), r=0,1, \ldots n\right\}_{n=0}^{\infty}$ is orthogonal over $T$ with respect to the weight function $W(u, v, w)=1$ if all the leading coefficients are positive and

$$
\iint_{T} W(u, v, w) \mathcal{P}_{n, r}(u, v, w) \mathcal{P}_{m, s}(u, v, w) d A=\delta_{n, m} \delta_{r, s}
$$

In the following section, the Legendre weight function $W(u, v, w)=1$ is considered.

\section{Legendre-Weighted Orthogonal Polynomials}

Farouki, Goodman, and Sauer determined in [5] closed-form representation of degree-ordered system of Legendre-weighted orthogonal polynomials $\mathcal{P}_{n, r}(u, v, w), r=0,1, \ldots, n, n \geq 0$ on the triangular domain $T=\{(u, v, w: u, v, w \geq 0, u+v+w=1\}$. These orthogonal polynomials are orthogonal with respect to the weight function $W(u, v, w)=1$. Since the Bernstein polynomials are stable, it is convenient to write these polynomials in Bernstein form. These polynomials $\mathcal{P}_{n, r}(u, v, w)$ are orthogonal to each polynomial of degree $\leq n-1$ and also orthogonal to each polynomial $\mathcal{P}_{n, s}(u, v, w), r \neq s$. They are given explicitly by:

$$
\mathcal{P}_{n, r}(u, v, w)=\sum_{i=0}^{r}(-1)^{r+i}\left(\begin{array}{c}
r \\
i
\end{array}\right)^{2} u^{i} v^{r-i} \sum_{j=0}^{n-r}(-1)^{j}\left(\begin{array}{c}
n+r+1 \\
j
\end{array}\right) b_{j}^{n-r}(w) .
$$


This formula is further simplified in [5] to:

$$
\mathcal{P}_{n, r}(u, v, w)=L_{r}\left(\frac{u}{1-w}\right)(1-w)^{r} q_{n, r}(w), r=0, \ldots, n,
$$

where $L_{r}, r=0,1, \ldots, n$ are the Legendre polynomials of degree $r$ and

$$
q_{n, r}(w)=\sum_{j=0}^{n-r}(-1)^{j}\left(\begin{array}{c}
n+r+1 \\
j
\end{array}\right) b_{j}^{n-r}(w), n \geq 0, r=0,1, \ldots, n .
$$

Traditionally, univariate Legendre polynomials are computed using different methods. The most convenient method is the recurrence relation in Equation (2). The method of construction in [5] is complicated and time-consuming, since many computations are required. Hence, there is a need for a simple evaluation method, which we present in the next section.

\section{Recurrence Relation}

Any three consecutive univariate orthogonal polynomials are related by a recurrence relation. In this section, it is shown that the bivariate orthogonal polynomials in Equation (5) also possess a recurrence relation. Any bivariate orthogonal polynomial from the $k$ th row is related by a recurrence relation to two orthogonal polynomials from the preceding two rows for all $r \neq n-1$. If $r=n-1$, then $\mathcal{P}_{n, n-1}(u, v, w)$ is related by a recurrence relation to two orthogonal polynomials from the $k-2$ nd and $k-3$ rd rows. The exact formulas are given in the following theorem.

Theorem 1. The bivariate orthogonal polynomials $\mathcal{P}_{n, r}(u, v, w), r=0,1, \ldots, n$ given by Equation (5) are computed using the following recurrence relations:

$$
\begin{aligned}
& \mathcal{P}_{n, r}(u, v, w)= \\
& \begin{cases}\frac{2 n-1}{n}(2 u+w-1) \mathcal{P}_{n-1, n-1}(u, v, w)-\frac{n-1}{n}(1-w)^{2} \mathcal{P}_{n-2, n-2}(u, v, w), & r=n \\
\left(\frac{2 n-3}{n-1}(2 u+w-1) \mathcal{P}_{n-2, n-2}(u, v, w)-\frac{n-2}{n-1}(1-w)^{2} \mathcal{P}_{n-3, n-3}(u, v, w)\right)(1-(2 n+1) w), & r=n-1 \\
\left(\alpha_{n, r}(1-2 w)-\beta_{n, r}\right) \mathcal{P}_{n-1, r}(u, v, w)-\gamma_{n, r} \mathcal{P}_{n-2, r}(u, v, w), & r \leq n-2\end{cases}
\end{aligned}
$$

where

$$
\alpha_{n, r}=\frac{n(2 n+1)}{(n-r)(n+r+1)}, \beta_{n, r}=\frac{n(2 r+1)^{2}}{(n-r)(n+r+1)(2 n-1)}, \gamma_{n, r}=\frac{(n-r-1)(n+r)(2 n+1)}{(n-r)(n+r+1)(2 n-1)} .
$$

and

$P_{0,0}(u, v, w)=1$

$P_{1,0}(u, v, w)=1-3 w, \quad P_{1,1}(u, v, w)=u-v$.

Proof. The construction of $\mathcal{P}_{n, r}(u, v, w)$ suggests that the recurrence relation depends on the values of $r$. Therefore, we have to distinguish between the three cases $r=n, r=n-1,0 \leq r \leq n-2$. In all cases, we begin with Equation (6) and do the proper arrangements and implications to get the required recurrence relations as follows:

The case $r=n$ :

We begin by substituting $r=n$ in Equation (6) to get

$$
\mathcal{P}_{n, n}(u, v, w)=L_{n}\left(\frac{u}{1-w}\right)(1-w)^{n} q_{n, n}(w) .
$$


Using the Definition (7) of $q_{n, r}(w)$ gives $q_{n, n}(w)=1$, leading to

$$
\mathcal{P}_{n, n}(u, v, w)=L_{n}\left(\frac{u}{1-w}\right)(1-w)^{n} .
$$

Substituting the recurrence Relation (2) of the Legendre polynomials in the last equation gives

$$
\mathcal{P}_{n, n}(u, v, w)=\left(\frac{2 n-1}{n}\left(\frac{2 u}{1-w}-1\right) L_{n-1}\left(\frac{u}{1-w}\right)-\frac{n-1}{n} L_{n-2}\left(\frac{u}{1-w}\right)\right)(1-w)^{n} .
$$

The last equation can be written in the form:

$$
\mathcal{P}_{n, n}(u, v, w)=\frac{2 n-1}{n}(2 u+w-1) L_{n-1}\left(\frac{u}{1-w}\right)(1-w)^{n-1}-\frac{n-1}{n}(1-w)^{2} L_{n-2}\left(\frac{u}{1-w}\right)(1-w)^{n-2} .
$$

Applying Equation (8) twice gives the required result.

The case $r=n-1$ :

We begin by substituting $r=n-1$ in Equation (6) to get

$$
\mathcal{P}_{n, n-1}(u, v, w)=L_{n-1}\left(\frac{u}{1-w}\right)(1-w)^{n-1} q_{n, n-1}(w) .
$$

Using the Definition (7) of $q_{n, n-1}(w)$ gives $q_{n, n-1}(w)=1-(2 n+1) w$. Substituting this equality and the recurrence Relation (2) for $L_{n-1}\left(\frac{u}{1-w}\right)$ in Equation (9) leads to:

$$
\mathcal{P}_{n, n-1}(u, v, w)=\left(\frac{2 n-3}{n-1}\left(\frac{2 u}{1-w}-1\right) L_{n-2}\left(\frac{u}{1-w}\right)-\frac{n-2}{n-1} L_{n-3}\left(\frac{u}{1-w}\right)\right)(1-w)^{n-1}(1-(2 n+1) w) .
$$

This is further simplified to:

$$
\begin{aligned}
\mathcal{P}_{n, n-1}(u, v, w)= & \left(\frac{2 n-3}{n-1}(2 u+w-1) L_{n-2}\left(\frac{u}{1-w}\right)(1-w)^{n-2}-\frac{n-2}{n-1}(1-w)^{2} L_{n-3}\left(\frac{u}{1-w}\right)(1-w)^{n-3}\right) \\
& (1-(2 n+1) w),
\end{aligned}
$$

Applying Equation (8) twice we get the required result. If either of the subscripts of $\mathcal{P}_{k, l}(u, v, w)$ is negative then the term $\mathcal{P}_{k, l}(u, v, w)$ is set to zero.

The case $0 \leq r \leq n-2$ :

We need to introduce the shifted univariate Jacobi polynomials $P_{n}^{(\alpha, \beta)}(u)$ of degree $n$ which are orthogonal on the interval $[0,1]$ with respect to the weight function $W(u)=u^{\alpha}(1-u)^{\beta}, \quad \alpha, \beta>-1$, see [1]. The following recurrence relation is the convenient method to compute the univariate Jacobi polynomials $P_{n}^{(\alpha, \beta)}(u)$ :

$$
P_{n}^{(\alpha, \beta)}(u)=\left(A_{n}(2 u-1)+B_{n}\right) P_{n-1}^{(\alpha, \beta)}(u)-C_{n} P_{n-2}^{(\alpha, \beta)}(u),
$$

where

$$
\begin{gathered}
A_{n}=\frac{(2 n+\alpha+\beta-1)(2 n+\alpha+\beta)}{2 n(n+\alpha+\beta)}, \\
B_{n}=\frac{\left(\alpha^{2}-\beta^{2}\right)(2 n+\alpha+\beta-1)}{2 n(n+\alpha+\beta)(2 n+\alpha+\beta-2)}, \\
C_{n}=\frac{(n+\alpha-1)(n+\beta-1)(2 n+\alpha+\beta)}{n(n+\alpha+\beta)(2 n+\alpha+\beta-2)} .
\end{gathered}
$$


Using Equation (4.3.2) in [1], we get the univariate Jacobi polynomials in terms of the Bernstein polynomials in the following form:

$$
P_{n}^{(\alpha, \beta)}(w)=\sum_{i=0}^{n}(-1)^{i} \frac{\left(\begin{array}{c}
n+\alpha \\
n-i
\end{array}\right)\left(\begin{array}{c}
n+\beta \\
i
\end{array}\right)}{\left(\begin{array}{c}
n \\
n-i
\end{array}\right)} b_{n-i}^{n}(w), n \geq 0, w \in[0,1] .
$$

Since $b_{n-i}^{n}(w)=b_{i}^{n}(1-w)$ and substituting $\alpha=0, \beta=2 r+1, n=n-r$, we get

$$
q_{n, r}(w)=P_{n-r}^{(0,2 r+1)}(1-w),
$$

and thus $q_{n, r}(w)$ satisfies the following recurrence relation:

$$
\begin{aligned}
q_{n, r}(w) & =\left(\frac{n(2 n+1)}{(n-r)(n+r+1)}(1-2 w)-\frac{n(2 r+1)^{2}}{(n-r)(n+r+1)(2 n-1)}\right) P_{n-r-1}^{(0,2 r+1)}(1-w) \\
& -\frac{(n-r-1)(n+r)(2 n+1)}{(n-r)(n+r+1)(2 n-1)} P_{n-r-2}^{(0,2 r+1)}(1-w) .
\end{aligned}
$$

Substituting the last relation in Equation (6) gives:

$$
\begin{aligned}
\mathcal{P}_{n, r}(u, v, w) & =L_{r}\left(\frac{u}{1-w}\right)(1-w)^{r}\left(\left(\frac{n(2 n+1)(1-2 w)}{(n-r)(n+r+1)}-\frac{n(2 r+1)^{2}}{(n-r)(n+r+1)(2 n-1)}\right) P_{n-r-1}^{(0,2 r+1)}(1-w)\right. \\
& \left.-\frac{(n-r-1)(n+r)(2 n+1)}{(n-r)(n-r+1)(2 n-1)} P_{n-r-2}^{(0,2 r+1)}(1-w)\right) .
\end{aligned}
$$

This is further simplified to:

$$
\begin{aligned}
\mathcal{P}_{n, r}(u, v, w) & =L_{r}\left(\frac{u}{1-w}\right)(1-w)^{r}\left(\frac{n(2 n+1)(1-2 w)}{(n-r)(n+r+1)}-\frac{n(2 r+1)^{2}}{(n-r)(n+r+1)(2 n-1)}\right) P_{n-r-1}^{(0,2 r+1)}(1-w) \\
& -L_{r}\left(\frac{u}{1-w}\right)(1-w)^{r} \frac{(n-r-1)(n+r)(2 n+1)}{(n-r)(n+r+1)(2 n-1)} P_{n-r-2}^{(0,2 r+1)}(1-w) .
\end{aligned}
$$

Substituting $\alpha=0, \beta=2 r+1, n=n-r-1$ in Equation (11), we get the following two equations:

$$
q_{n-1, r}(w)=P_{n-r-1}^{(0,2 r+1)}(1-w), \quad q_{n-2, r}(w)=P_{n-r-2}^{(0,2 r+1)}(1-w) .
$$

Thus the last equation for $\mathcal{P}_{n, r}(u, v, w)$ is simplified to

$$
\begin{aligned}
\mathcal{P}_{n, r}(u, v, w) & =L_{r}\left(\frac{u}{1-w}\right)(1-w)^{r}\left(\frac{n(2 n+1)(1-2 w)}{(n-r)(n+r+1)}-\frac{n(2 r+1)^{2}}{(n-r)(n+r+1)(2 n-1)}\right) q_{n-1, r}(w) \\
& -L_{r}\left(\frac{u}{1-w}\right)(1-w)^{r} \frac{(n-r-1)(n+r)(2 n+1)}{(n-r)(n+r+1)(2 n-1)} q_{n-2, r}(w) .
\end{aligned}
$$

Applying Equation (8) twice, we get the required result. This completes the proof of the Theorem.

\section{Conclusions}

In this paper, recurrence relations for the Legendre-weighted orthogonal polynomials $\mathcal{P}_{n, r}(u, v, w), r=0,1, \ldots, n, n \geq 0$ on the triangular domain $T=\{(u, v, w): u, v, w \geq 0, u+v+w=1\}$ are presented. Unlike the method of construction in [5], our recursive algorithm enables us to compute these polynomials efficiently in a very simple fashion. 
Acknowledgments: The author owes thanks to the reviewers for helpful and invaluable comments and suggestions for improving an earlier version of this manuscript.

Conflicts of Interest: The author declares no conflict of interest.

\section{References}

1. Szegö, G. Orthogonal Polynomials, 4th ed.; American Mathematical Society: Providence, RI, USA, 1975.

2. Dunkl, C.F.; Xu, Y. Orthogonal Polynomials of Several Variables (Encyclopedia of Mathematics and Its Applications); Cambridge University Press: Cambridge, UK, 2002.

3. Suetin, P.K. Orthogonal Polynomials in Two Variables; Gordon and Breach: Amsterdam, The Netherlands, 1999.

4. Sauer, T. The genuine Bernstein-Durrmeyer operator on a simplex. Results Math. 1994, 26, 99-130.

5. Farouki, R.T.; Goodman, T.N.T.; Sauer, T. Construction of orthogonal bases for polynomials in Bernstein form on triangular and simplex domains. Comput. Aided Geom. Des. 2003, 20, 209-230.

6. Lewanowicz, S.; Wozńy, P. Connections between two-variable Bernstein and Jacobi polynomials on the triangle. J. Comput. Appl. Math. 2006, 197, 520-533.

7. Area, I.; Godoy, E.; Ronveaux, A.; Zarzo, A. Bivariate second-order linear partial differential equations and orthogonal polynomial solutions. J. Math. Anal. Appl. 2012, 387, 1188-1208.

8. Höllig, K.; Hörner, J. Approximation and Modeling with B-Splines; Society for Industrial and Applied Mathematics (SIAM): Philadelphia, PA, USA, 2013.

9. Khan, S.; Al-Gonah, A.; Yasmin, G. Generalized and mixed type Gegenbauer polynomials. J. Math. Anal. Appl. 2012, 390, 197-207.

10. Pathan, M.; Khan, W. A New class of generalized polynomials associated with Hermite and Euler polynomials. Mediterr. J. Math. 2015, doi:10.1007/s00009-015-0551-1.

11. Cooper, S.; Waldron, S. The diagonalisation of multivariate Bernstein operator. J. Approx. Theory 2002, 117, 103-131.

12. Derriennic, M. On multivariate approximation by Bernstein-type polynomials. J. Approx. Theory 1985, 45, 155-166.

13. Waldron, S. A generalised beta integral and the limit of the Bernstein-Durrmeyer operator with Jacobi weights. J. Approx. Theory 2003, 122, 141-150.

14. Rababah, A. Distance for degree raising and reduction of triangular Bézier surfaces. J. Comput. Appl. Math. 2003, 158, 233-241.

(C) 2016 by the author; licensee MDPI, Basel, Switzerland. This article is an open access article distributed under the terms and conditions of the Creative Commons by Attribution (CC-BY) license (http://creativecommons.org/licenses/by/4.0/). 